\title{
Faisabilité d'une méthode d'évaluation de la résistance thermique de contact entre une lamelle céramique écrasée sur un substrat métallique
}

\author{
A. Grimaud ${ }^{a}$, M. Bouneder, S. Menecier et M. ElGanaoui \\ Sciences des Procédés Céramiques et Traitements de surface (SPCTS) \\ Université de Limoges CNRS UMR 6638, 123 avenue Albert Thomas, 87060 Limoges Cedex, France
}

\begin{abstract}
Résumé - On se propose d'évaluer une procédure de détermination expérimentale de la Résistance Thermique de Contact (RTC) entre une lamelle de céramique (de $1 \mu \mathrm{m}$ d'épaisseur) obtenue par projection plasma et un substrat métallique lisse. La procédure consiste à soumettre la lamelle à un flux thermique et à déterminer expérimentalement le temps nécessaire à sa fusion et à effectuer le calcul du champ de température de la configuration dépôt-substrat, paramétré par la valeur de la RTC. Le flux thermique est apporté par un faisceau laser et la durée d'irradiation de la lamelle est donnée par la vitesse de déplacement de ce faisceau. Des résistances thermiques de contact de l'ordre de $10^{-4} \mathrm{~m}^{2} . \mathrm{K} / \mathrm{W}$ ont été mesurées, ce qui étend la gamme des méthodes d'identification des RTC aux grandes valeurs.
\end{abstract}

Mots clés : Résistance Thermique de Contact (RTC) / projection plasma / fusion

\begin{abstract}
Faisabilité d'une méthode d'évaluation de la résistance thermique de contact entre une lamelle céramique écrasée sur un substrat métallique. This paper presents an experimental procedure to evaluate the Thermal Contact Resistance (RTC) between a ceramic deposit (of $1 \mu \mathrm{m}$ in thickness), obtained by plasma spraying, and metallic polish substrate. The procedure consists on submitting the lamella to a heat flux and calculates the thermal field in the deposit/substrate configuration taking into account the RTC parameter. Heat flux is provided by a laser beam and the lamella irradiation duration is estimated from the laser scanning velocity. Values of $10^{-4} \mathrm{~m}^{2} . \mathrm{K} / \mathrm{W}$ of the RTC have been measured, that extend the identification range with the present method to strong values of this parameter.
\end{abstract}

Key words: Thermal Contact Resistance / plasma spraying / melting

\section{Introduction}

Les mécanismes de croissance et l'adhésion d'un dépôt sont étroitement liés aux propriétés physiques et chimiques superficielles du support (substrat). La détermination de la résistance thermique d'interface peut éclaircir certains aspects de ce couplage en caractérisant la présence d'impuretés, de fissures ou de décollements liés au procédé d'élaboration.

Une méthode de mesure de la résistance d'interface entre un dépôt micronique et un substrat métallique est présentée. Elle est basée sur l'identification thermodynamique du changement de phase solide-liquide à partir de la réponse à l'échauffement produit par une impulsion laser. De fortes résistances thermiques peuvent être détectées (de l'ordre de $10^{-4} \mathrm{~m}^{2} \mathrm{~K} / \mathrm{W}$ )

\footnotetext{
a Auteur correspondant : alain.grimaud@unilim.fr
}

lorsque l'observation de la fusion s'effectue dans les premières dizaines de milliseconde. Cette technique complète la gamme d'estimation de la RTC pour des valeurs importantes [1].

\section{Expérimentations}

La phase expérimentale comprend deux étapes principales : la réalisation des lamelles, et le procédé de fusion.

\subsection{Réalisation des lamelles}

La poudre utilisée est une zircone yttriée fondue broyée de granulométrie moins de $45 \mu \mathrm{m}$ et plus de $22 \mu \mathrm{m}$. Plusieurs substrats métalliques sont testés : Acier inoxydable (304L), cuivre, alliage d'aluminium (AU4G), et fonte lamellaire (FT25). 


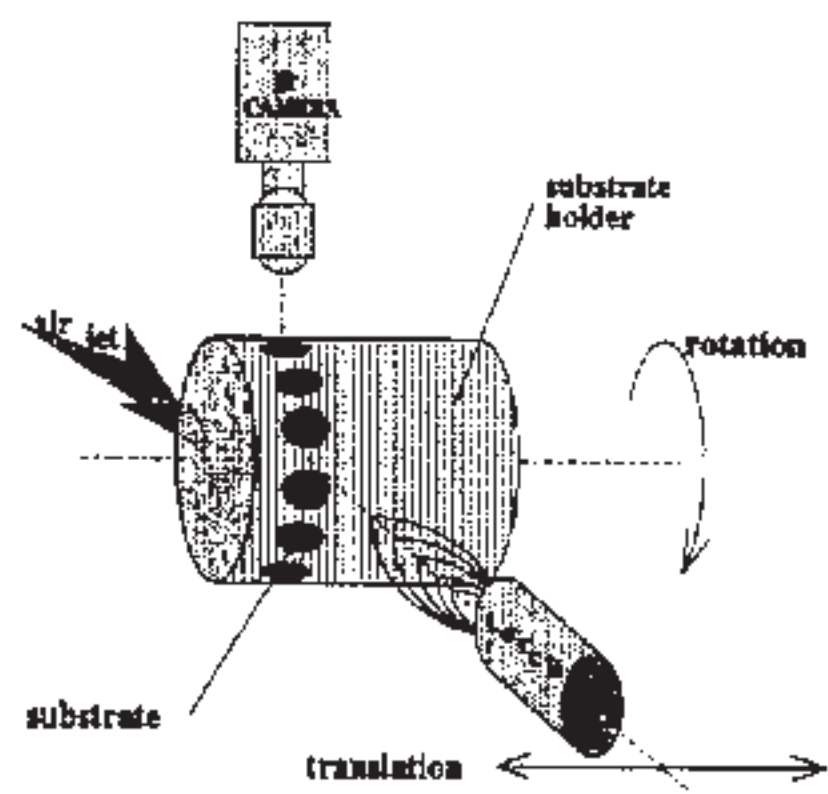

Fig. 1. Schéma de principe d'un équipement de projection thermique.

Les substrats sont polis par papier $\mathrm{SiC}$ (jusqu'au grade 4000) pour obtenir une rugosité correspondant à un Ra de $0.1 \mu \mathrm{m}$ puis dégraissés à l'acétone avant le tir. Les substrats sont préchauffés par la flamme plasma jusqu'à $200{ }^{\circ} \mathrm{C}$ pour la fonte, le cuivre et l'aluminium et jusqu'à $300{ }^{\circ} \mathrm{C}$ pour l'acier inoxydable.

Dans ces conditions, le substrat en fonte subit une oxydation de surface importante [2] alors que ceux en acier ou en cuivre ne sont que très légèrement oxydés.

On considère que la température de préchauffage est atteinte lorsque, compte tenu des déplacements relatifs torche-pièce, la température lue sur un pyromètre embarqué sur un porte-échantillons visant un point de l'anneau porte échantillons à 60 degrés avant l'impact des poudres (et du jet de plasma) est égale à la valeur donnée pour le préchauffage. En pratique, la température de ce point varie avec une amplitude de l'ordre de $50{ }^{\circ} \mathrm{C}$, selon la masse des échantillons, de leur support et des déplacements de la torche. La température prise en compte par l'opérateur pour le préchauffage est égale dans nos conditions expérimentales à $200^{\circ} \mathrm{C} \pm 20^{\circ} \mathrm{C}$ (pour la fonte et l'alumine) et à $300{ }^{\circ} \mathrm{C} \pm 30^{\circ} \mathrm{C}$ (pour l'acier inoxydable).

Les tirs se font à l'air et les échantillons sont disposés sur un cylindre (Fig. 1).

On introduit une poudre de zircone yttriée (partiellement stabilisée avec 7\% d'yttrine) dans un jet de plasma d'arc soufflé (argon $45 \mathrm{Nl} / \mathrm{min}$, hydrogène $15 \mathrm{Nl} / \mathrm{min}$, 600 ampères, 65 volts). La distance de tir est de $100 \mathrm{~mm}$.

Dans ces conditions, on obtient après tir des grains, des lamelles écrasées « splats ». La morphologie des splats est illustrée sur la Figure 2. L'épaisseur des splats mesurée par palpeur est de l'ordre de $1 \mu \mathrm{m}$ (un exemple de profil est donnée à la Fig. 3).

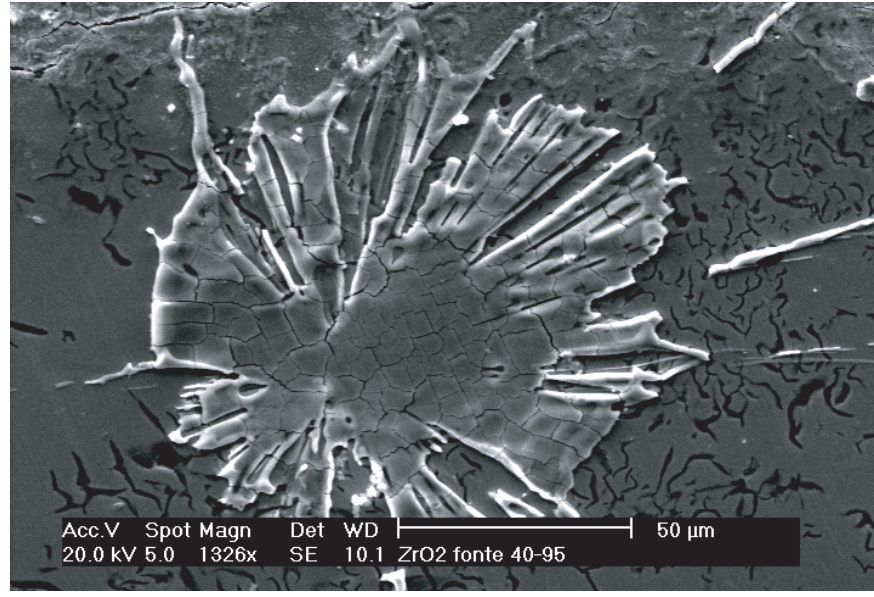

Fig. 2. Splat de zircone yttriée sur substrat en fonte.

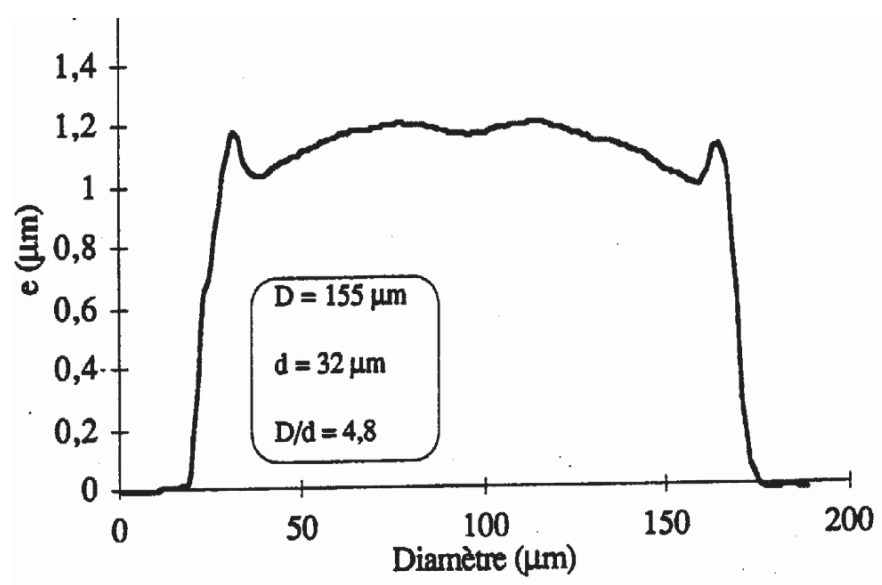

Fig. 3. Profil écrasement d'un grain de diamètre initial $32 \mu \mathrm{m}$.

C'est cette valeur arrondie qui est utilisée pour les calculs numériques, la recherche de valeurs exactes pour chaque grain ne semble pas justifiée à ce niveau du travail.

\subsection{Refusion des lamelles}

Un laser continu à fibre double gaine (YLR IPG Photonics) de puissance maximale de $100 \mathrm{~W}$ et émettant dans le proche infrarouge (1072 nm) est utilisé. Le profil d'intensité du faisceau est Gaussien $\left(\mathrm{TEM}_{00}\right)$. Son diamètre $2 \omega_{0}$, définit comme la largeur du faisceau pour les valeurs d'intensité supérieures à $I_{0} / e^{2}$ (où $I_{0}$ est l'intensité maximale au centre variant entre 0,7 et $5 \mathrm{~mm})$. Ce laser est associé à une table XY permet des vitesses de déplacement faisceau jusqu'à $100 \mathrm{~mm} / \mathrm{s}$ (Fig. 4).

L'intensité $I(x, y)$ du faisceau laser est donnée par la relation (1):

$$
I(x, y)=\frac{2 P}{\pi \omega_{0}^{2}} \exp \left(\frac{-2\left(x^{2}+y^{2}\right)}{\omega_{0}^{2}}\right)
$$

où $P$ est la puissance du laser appliquée.

L'énergie effectivement apportée par le laser lors du balayage dépend de la vitesse $v$ selon un seul axe. Si 


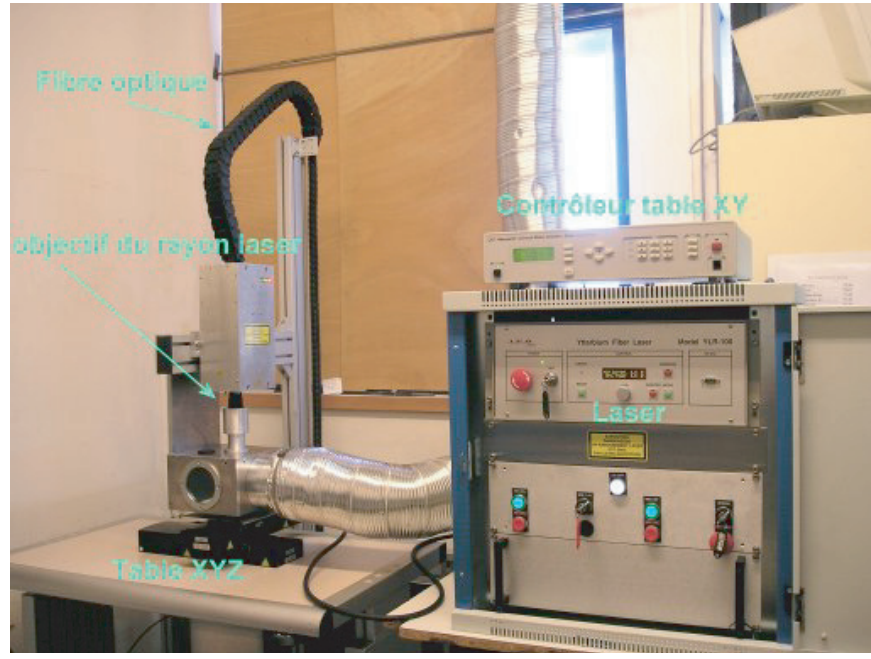

Fig. 4. Équipement laser et dispositif de balayage.

le laser se déplace selon l'axe $y$, la fonction représentant l'énergie surfacique $E(x, y)$ apportée en chaque point du substrat est donnée par la relation (2). Le calcul est limité à un déplacement de quatre diamètres de spot.

$$
E(x, y)=\int_{\frac{-4 \omega_{0}}{v}}^{\frac{4 \omega_{0}}{v}} I(x, y+v t) \mathrm{d} t
$$

Cette énergie disponible n'est que partiellement absorbée par l'échantillon. L'énergie effectivement disponible pour le substrat dépend de son coefficient d'absorption A à la longueur d'onde du laser. L'énergie surfacique absorbée Ea $(0,0)$ par le substrat au centre du rayon laser est :

$$
E_{a}(0,0)=\frac{2 A P}{\sqrt{2 \pi} \omega_{0} v}
$$

où $P$ est la puissance du laser et $\nu$ est sa vitesse de déplacement. Grâce à cet équipement, les échantillons portant les «splat» peuvent êtres balayé dans la gamme de vitesse de $0.01 \mathrm{~mm} / \mathrm{s}$ à $100 \mathrm{~mm} / \mathrm{s}$ et de flux allant jusqu'à $100 \mathrm{~W}$.

Pour évaluer le flux thermique réel apporté aux grains nous avons recouvert un bloc de cuivre $(10 \times 10 \times 10 \mathrm{~mm})$ d'une quasi monocouche de grains écrasés et soumis cette face au faisceau laser en déplacement durant $10 \mathrm{~s}$, tout en mesurant l'élévation de température de celui-ci par thermocouple placé en son centre. Les pertes thermiques latérales sont négligées (faibles devant la totalité du flux absorbé par la surface). Pour les conditions de puissance et de taille de spot utilisé par la suite, le flux absorbé est considéré égal à $30 \%$ du flux incident. Cette valeur sera utilisée pour la modélisation numérique.

Rappelons que l'on recherche des seuils du couple (vitesse, flux thermique), sous contrainte de la fusion du splat (sans le substrat). Les essais préliminaires pour chaque échantillon, ont conduit à fixer le flux à une valeur de $95 \mathrm{~W}$. Les tests se font en opérant sur l'échantillon des traces parallèles pour différentes vitesses. A vitesse élevée,

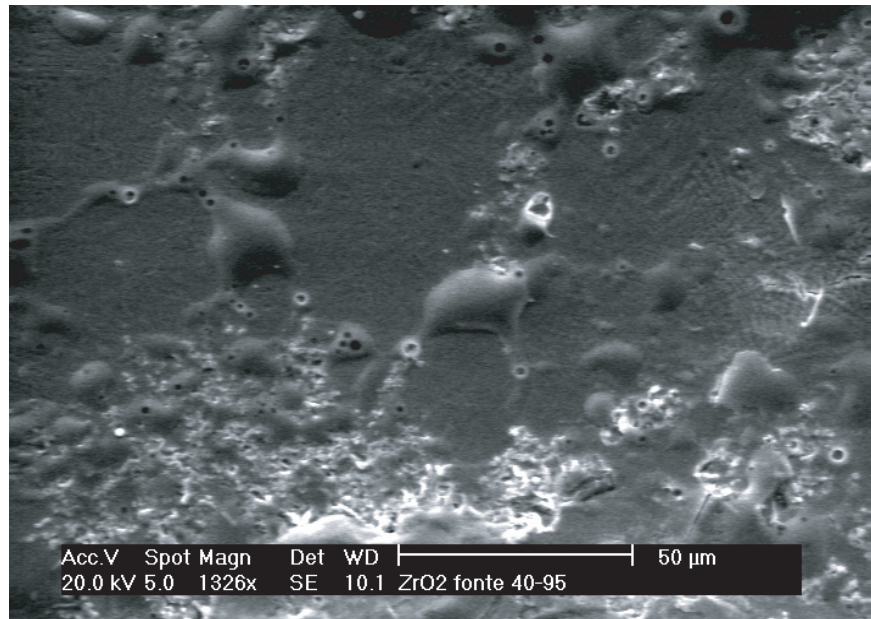

Fig. 5. Absence de refusions dans la zone exposée au faisceau laser (zircone sur acier).

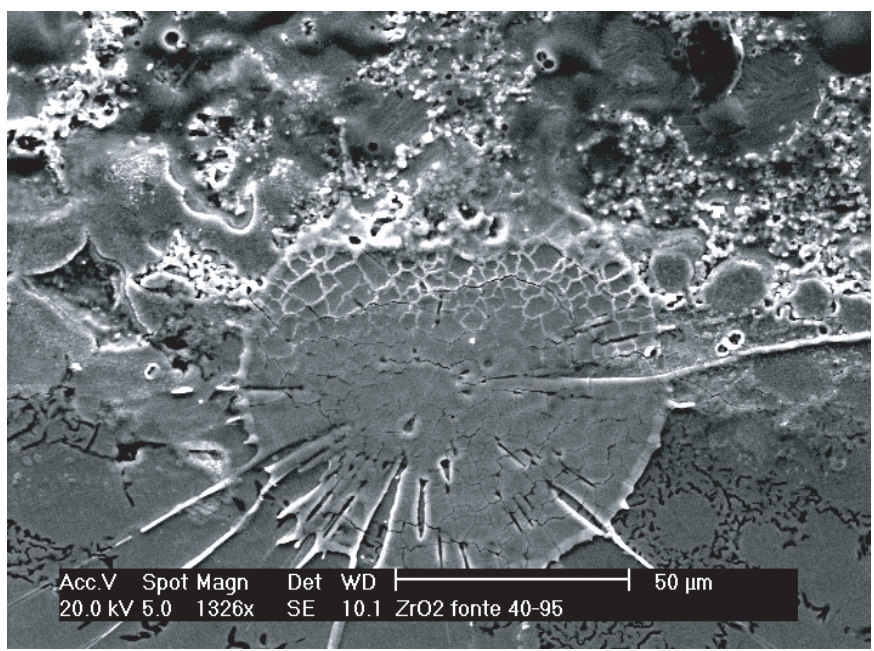

Fig. 6. Refusions dans la zone exposée au faisceau laser (zircone sur fonte).

rien ne se passe (Fig. 5). A faible vitesse tout peut fondre (support et dépôt), Figure 6. La vitesse pour laquelle les grains fondent seuls permet de donner la durée d'irradiation à partir d'un examen en microscopie optique et électronique des grains fondus. Les essais laser ont révélé que les paramètres optimaux pour faire fondre des couches déposée (splats) de $60 \mu \mathrm{m}$ de diamètre en zircone sur de la fonte lamellaire FT25 sont une puissance de $95 \mathrm{~W}$ à une vitesse de $40 \mathrm{~mm} / \mathrm{s}$.

Cette durée est ensuite exploitée dans un modèle de calcul comportant outre les propriétés thermiques des matériaux, des valeurs variables de la RTC.

A travers le modèle de calcul, le temps conduisant à la fusion donné par l'expérience, permet de remonter à la bonne valeur de la résistance thermique de contact. 


\section{Evaluation de la RTC}

\subsection{Principe de la méthode}

Le modèle numérique permet d'accéder à partir des données expérimentales (vitesse, flux, durée d'irradiation, ...) à l'estimation des valeurs de la RTC favorables à la fusion. Ainsi la détermination des transferts thermiques dans le système splat-substrat permet de quantifier exactement la valeur de la RTC. Une géométrie 1D est utilisée, car les pertes convectives et radiatives relatives aux faces supérieure et latérales sont négligeables. Compte tenu de l'échelle de temps très courte de la phase de chauffage et des dimensions spatiales de la surface d'échange de la lamelle, le transfert de chaleur est considéré purement diffusif dans un milieu homogène et isotrope bicouche en contact imparfait. Les propriétés thermo-physiques des matériaux sont considérées constantes.

Le modèle de transfert de chaleur associée à la couche $i$, aux conditions initiales et aux limites ainsi qu'aux conditions de contact entre couches est considéré :

$$
\begin{aligned}
& \frac{\partial T_{j}}{\partial^{t}}=\alpha_{j} \frac{\partial^{2} T_{j}}{\partial z^{2}} \quad j=a, b \\
& \grave{a} \quad t=0 \quad T_{a}(z, 0)=T_{b}(z, 0)=T_{i} \\
& \text { en } \quad z=0 \quad-k_{a} \frac{\partial T_{a}}{\partial z}=q_{\text {laser }}^{\prime \prime} \\
& \text { en } \quad z=L \quad\left[k_{b} \frac{\partial_{b}^{T}}{\partial^{z}}\right]=0
\end{aligned}
$$

A l'interface entre deux matériaux a et b :

$$
\left[k_{a} \frac{\partial T_{a}}{\partial z}\right]_{\Sigma}=\left[k_{b} \frac{\partial T_{a}}{\partial z}\right]_{\Sigma}=\frac{-\Delta T}{R T C}
$$

où $\Delta T=T_{\Sigma}^{b}-T_{\Sigma}^{a}$ est le saut le température à l'interface.

\subsection{Analyse des résultats}

La résolution numérique de ce problème thermique multicouche à travers une approximation numérique de type différences finies implicites est décrite et validée en [3]. Dans le cas ou l'on néglige le splat de zircone, la longueur de relaxation pour ce système semi infini est $L=\sqrt{2 \alpha t}$. Le modèle de calcul a été ainsi validé en comparant les résultats obtenus à ceux d'une solution analytique, dans le cas d'une impulsion laser opérant en $z=0$ sur un domaine semi infini à propriétés physiques constantes et à température initiale nulle [4] :

$$
\theta=\frac{2 q_{\text {laser }}^{\prime \prime}}{k}\left[\left(\frac{\alpha t}{\pi}\right)^{1 / 2} \exp \left(\frac{-z^{2}}{4 \alpha t}\right)-\frac{z}{2} \operatorname{erfc} \frac{z}{2 \sqrt{\alpha t}}\right]
$$

Pour un coefficient d'absorption $A=0.3$, le splat de zircone, reçoit une fluence $1.263310^{+08} \mathrm{~W} / \mathrm{m}^{2}$ et fond au bout de $13.4 \mathrm{~ms}$. Les prévisions du modèle numérique montrent alors que cela correspond à une RTC de l'ordre de $7 \times 10^{-5} \mathrm{~m}^{2} . \mathrm{K} / \mathrm{W}$ comme le montre la figure 7 . Cette

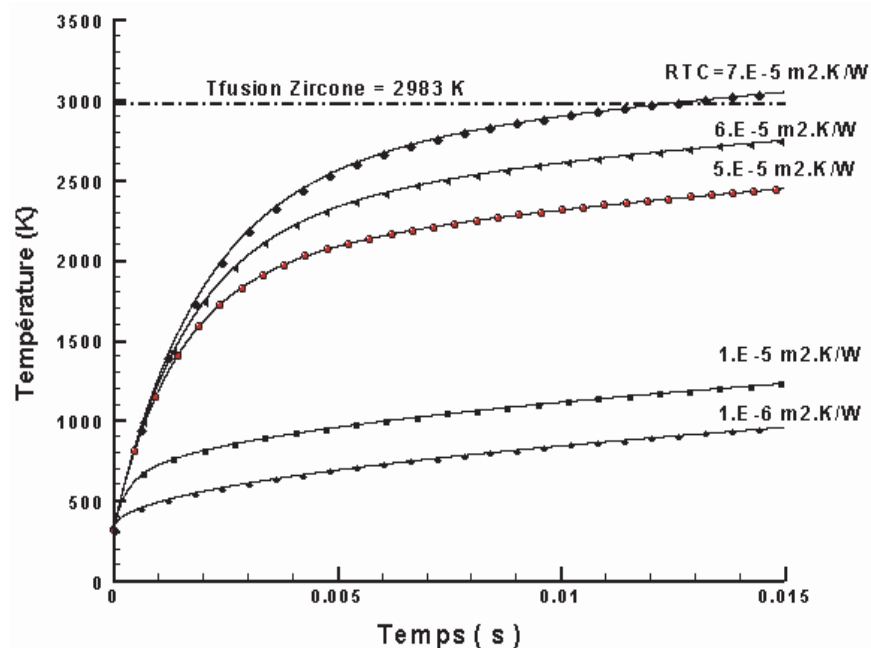

Fig. 7. Évolution de température au milieu du splat en fonction de la RTC.

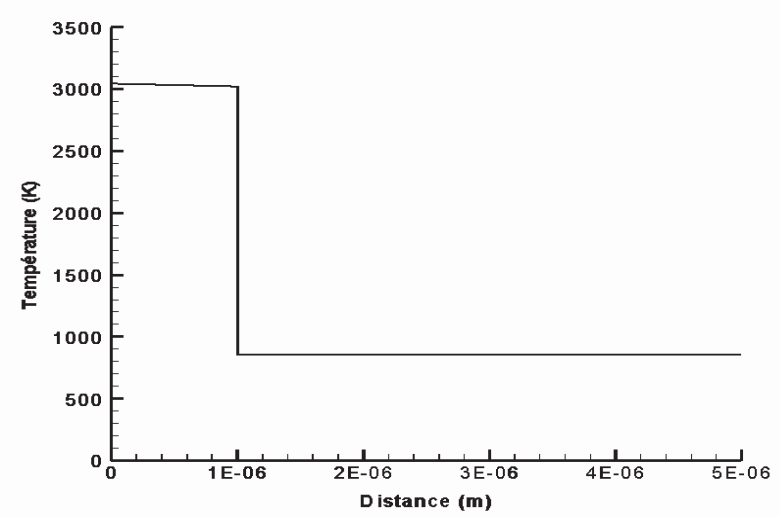

Fig. 8. Distribution de température en configuration lamelle substrat au bout de 0.015 secondes.

valeur reste cependant élevée, comparativement à celles communément admises dans les expériences de projection thermique (entre $1 \times 10^{-6}$ et $\left.1 \times 10^{-8} \mathrm{~m}^{2} . \mathrm{K} / \mathrm{W}\right)$ [5], et ceci suggère que les splats de zircone sont peu adhérents dans ce cas, à cause de la présence d'une couche poreuse et isolante d'oxyde de fer $\mathrm{Fe}_{2} \mathrm{O}_{3}$ (hématite), formée durant la phase de préchauffage. Sous des conditions d'expérimentation identiques à celles que nous avons utilisé sur un substrat de fonte, les essais sur du cuivre ou sur de l'acier inox, ne nous ont pas permis d'observer la fusion des lamelles de zircone. Les prévisions numériques pour ces substrats montrent que la résistance thermique de contact a une valeur inférieure à celle précédemment trouvée. Cette valeur de la RTC, induit effectivement un saut de température à l'interface lamelle-substrat très élevé (de l'ordre $2100 \mathrm{~K}$ comme le montre la Fig. 8).

Pour un substrat en inox, la RTC ne dépasse pas la valeur de $5 \times 10^{-5} \mathrm{~m}^{2}$.K/W alors que si elle avait été de $6 \times 10^{-5} \mathrm{~m}^{2} . \mathrm{K} / \mathrm{W}$, on aurait observé une fusion de la lamelle de zircone. Pour le cas du cuivre on a une valeur de 


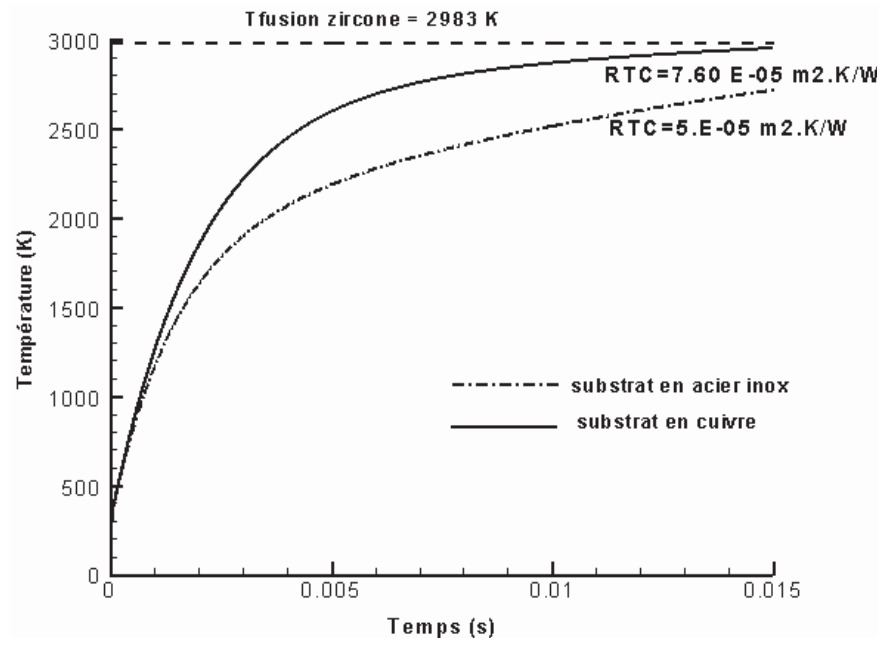

Fig. 9. Évolution de température d'une lamelle de zircone sur un substract de cuivre ou d'acier inox.

la RTC inférieure à $7.6 \times 10^{-5} \mathrm{~m} 2 . \mathrm{K} / \mathrm{W}$ (Fig. 9). On peut noter qu'en plus de la forte diffusivité de ces matériaux (considérés comme des puits de chaleur), vis-à-vis de la fonte, il faudrait un rayonnement très bien réfléchi par le métal poli mais également très bien absorbé par le grain écrasé. La valeur de la RTC dans ces configurations est donc plus faible et il convient d'appliquer des fluences bien plus élevées pour espérer une fusion des lamelles.

\section{Conclusion}

Cette étude montre la faisabilité d'une méthode expérimentale de mesure de la résistance thermique de contact entre des grains de céramique (à haut point de fusion) de l'ordre du micromètre d'épaisseur et un substrat métallique. Les prévisions numériques du modèle de calcul sont en parfait accord avec les observations expérimentales, et les valeurs des RTC que nous avons déterminées sont de l'ordre de $1 \times 10^{-4} \mathrm{~m}^{2} . \mathrm{K} / \mathrm{W}$.

La valeur élevée de la RTC trouvée dans le cas de la fonte est liée à la présence d'oxyde de fer $\mathrm{Fe}_{2} \mathrm{O}_{3}$ qui s'est formée au cours de la phase de préchauffage. Ce dernier bien que favorisant l'adhésion du splat au substrat, il induit une forte oxydation dans le cas de la fonte (faible pour un substrat de cuivre ou d'acier). La méthode proposée permet d'accéder à une estimation de fortes valeurs de la RTC; sa précision dépend cependant d'une bonne connaissance du coefficient d'absorption effective de la lamelle.

Remerciements. Le premier auteur souhaite remercier le programme Franco-algérien (FAD). Remerciements aux professeurs J.C. Labbe et P. Lefort pour la mise à disposition du Laser.

\section{Références}

[1] Lahmar, T.P. Nguyen, D. Sakami, S. Orain, Y. Scudeller, F. Danes, Experimental investigation on the thermal contact resistance between gold coating and ceramic substrates, Thin Solid Films, Volume 389, Issues 1-2, 15 June 2001, 167-172

[2] L. Bianchi. Thèse de doctorat, Université de Limoges (1999)

[3] M. Bouneder M., M. El Ganaoui, B. Pateyron, P. Fauchais, Thermal modelling of composite iron/alumina particles sprayed under plasma conditions part I : pure condition, Int. J. of High Temp. Mater. Proces. vol.7 (2003), 547-558

[4] H.S Carslaw, J.C. Jaeger, Conduction of heat in solids, Oxford University Press 1959

[5] P. Fauchais, M. Fukumoto, A. Vardelle, M. Vardelle, Knowledge concerning splat formation : An invited review, J. of Thermal Spray Technology, Vol. 13 (2004), 337-360 